\section{A note on delay coordinates for locally observable analytic systems}

\author{
Alberto Padoan and Alessandro Astolfi
}

\begin{abstract}
In this short note the problem of locally reconstructing the state of a nonlinear system is studied. To avoid computational difficulties arising in the numerical differentiation of the output, the so-called delay coordinates are considered. The assumptions of analyticity and (local) observability of the system are shown to imply that a family of mappings, induced by the delay coordinates and parameterized by a time delay parameter, gives a local diffeomorphism for generic values of such delay parameter on a certain set. A worked-out example illustrates the result.
\end{abstract}

\section{INTRODUCTION}

The construction of (local) state observers is of paramount importance in nonlinear control theory [1-3]. Local state observers are instrumental for the solution of a number of control problems, such as feedback stabilization, output regulation and fault detection, see, e.g., [4-8]. Most observer design techniques require the knowledge of finitely many high-order derivatives of the output, which must be computed at the same time instants [9]. In practice the time derivatives of the output are not always available as measured data, and these quantities have to be estimated by means of numerical differentiation algorithms [10]. This is often computationally difficult, reason being that numerical differentiation is an ill-conditioned problem. As a consequence the applicability of the obtained observers can be severely undermined by errors in the initial estimates and noise: small perturbations on the output measurements may induce large errors in its high-order time derivatives.

A different strategy to solve the problem of local state reconstruction can be considered. To circumvent the problem of approximating high-order time derivatives of the output, the state of a nonlinear system can be expressed as a function of the so-called delay coordinates, i.e. the output and finitely many delayed versions of it. Delay coordinates have received considerable attention in the field of mathematical physics over the past three decades. These objects have been studied in relation to embedding techniques for the reconstruction of attractors from observed time series. The most important result of this line of research have been given in [11 12]. Therein it has been shown that at most $2 n+1$ delay coordinates, where $n$ is the dimension of the state space, are needed to build vectors which lie generically on an embedded manifold of the original state space. This method of state space reconstruction is often referred to as the method of delays in literature. It should be acknowledged that the method of delays has led several authors to disregard the problem of estimating the state of the system, since vectors built with the method of delays describe the evolution on a state-space (diffeomorphically) equivalent to the original one. Similar results have been obtained in a probabilistic setting using the notion of prevalence in [13].

The approach proposed in this article is similar in spirit to the line of research mentioned above. A special mapping, induced by the delay coordinates vector and parameterized by the sampling period, is studied to solve this problem. Our main contribution is to show that such mapping defines a (local) diffeomorphism for generic

A. Padoan is with the Department of Electrical and Electronic Engineering, Imperial College London, London SW7 2AZ, UK (e-mail: alberto.padoan130imperial.ac.uk). A. Astolfi is with the Department of Electrical and Electronic Engineering, Imperial College London, London SW7 2AZ, UK and with the Dipartimento di Ingegneria Civile e Ingegneria Informatica, Università di Roma "Tor Vergata", Via del Politecnico 1, Rome 00133, Italy (e-mail:a.astolfi@imperial.ac.uk). values of the sampling period on a given domain. The assumptions of analyticity and (local) observability (see Section II for a formal definition) of the system are the price to be paid to obtain our results. The established result is illustrated with a simple academic example.

The rest of the article is organized as follows. Section $\Pi$ contains the problem formulation and introduces basic assumptions. Section III illustrates our main result, which implies that $n$ time delayed versions of the output of a (locally) observable system can be employed to construct local state observers for generic values of the time delay (in a set to be specified later). In Section IV our results are evaluated on a simple illustrative example. Finally, concluding remarks are given in Section $\mathrm{V}$

Notation: Standard notation is used. $\mathbb{R}, \mathbb{R}^{n}$ and $\mathbb{R}^{p \times m}$ denote the set of real numbers, of $n$-dimensional vectors with real entries, and of $p \times m$-dimensional matrices with real entries, respectively. The set of positive integers $\{i, i+1, \ldots, j\}$, with $0 \leq i \leq j$, is denoted by $[i, j]$. The flow of a smooth vector field $f$ is denoted by $\phi_{t}(x)$, so the curve $t \rightarrow \phi_{t}(x)$ is the integral curve of $f$ passing through $x$ at time $t=0$. The Lie derivative of the smooth function $h$ along the smooth vector field $f$ is defined as $L_{f} h=\frac{\partial h}{\partial x} f$, and the functions $L_{f}^{k} h$, with $k$ a non-negative integer, are defined recursively as $L_{f}^{k} h=L_{f}\left(L_{f}^{k-1} h\right)$, with $L_{f}^{0} h=h$. The differential of a smooth mapping $\varphi$ is denoted by $d \varphi$.

\section{Problem Formulation}

Consider a continuous-time, autonomous, nonlinear system described by equations of the form

$$
\dot{x}=f(x), \quad y=h(x),
$$

in which $x(t) \in \mathbb{R}^{n}$ and $y(t) \in \mathbb{R}$ denote the unmeasured state and the measured output of the system at time $t$, respectively. Assume, without loss of generality, that the state of the system evolves on an open connected set $\mathcal{X} \subseteq \mathbb{R}^{n}$ containing the initial condition for all $t \in \mathbb{R}$. Similar considerations can be performed when the state of the system is only defined on a real interval of the form $\left(t_{\min }, t_{\max }\right)$, with $t_{\min }>0$ and $t_{\max }>0$. The system is assumed to be analytic, i.e. the vector field $f: \mathcal{X} \rightarrow \mathcal{X}$ and the function $h: \mathcal{X} \rightarrow \mathbb{R}$ are real analytic functions of their arguments.

Goal of this paper is to demonstrate that, under certain assumptions, the so-called "delay coordinates" vector, defined as

$$
Y_{T}(t)=\left[\begin{array}{c}
y(t) \\
y(t+T) \\
\vdots \\
y(t+(n-1) T)
\end{array}\right],
$$

for all $t \in \mathbb{R}$, can be used to reconstruct (locally) the state of the system for generic values of $T$ in a real interval (to be specified later). Note that the delay coordinates are composed by the output signal and $n-1$ delayed versions of it. The parameter $T$ represents the sampling period, i.e. the time interval between two distinct samples of the output. Without loss of generality, $T$ is assumed to be a userdefined positive real number that can be assigned. This assumption is made for notational simplicity: changing the sign of $T$ causes the appearance of cumbersome minus signs in the following derivations.

To streamline the presentation of our results, we introduce the mapping $H_{T}: \mathcal{X} \rightarrow \mathbb{R}^{n}$, parameterized by the real parameter $T$, and defined as

$$
H_{T}(x)=\left[\begin{array}{c}
h(x) \\
h \circ \phi_{T}(x) \\
\vdots \\
h \circ \phi_{(n-1) T}(x)
\end{array}\right],
$$


for all $x \in \mathcal{X}$.

We now recall a notion of observability ${ }^{1}$ for nonlinear systems $\mid 1$. 3]. The observation space $\mathcal{O}$ of system (1) is the linear space over $\mathbb{R}$ of real analytic functions on $\mathcal{X}$ containing the function $h$ and all repeated Lie derivatives $L_{f}^{i} h$ for each integer $i>0$. The observation space $\mathcal{O}$ defines the observability codistribution

$$
d \mathcal{O}(x)=\operatorname{span}\{d \varphi(x), \varphi \in \mathcal{O}\}, x \in \mathcal{X} .
$$

System (1) is said to satisfy the observability rank condition at $x_{0} \in$ $\mathcal{X}$ if

$$
\operatorname{dim} d \mathcal{O}\left(x_{0}\right)=n
$$

Throughout the paper, system (1) is assumed to satisfy the observability rank condition at some given point $x_{0} \in \mathcal{X}$. A consequence of this assumption is that system (1) is (locally) observable [1]. In addition, if $x_{0}=x\left(t_{0}\right) \in \mathcal{X}$, then there exist a positive integer $k$, with $k \geq n$, and a mapping $\psi: \mathbb{R}^{k} \rightarrow \mathbb{R}^{n}$ such that $x_{0}=\psi\left(y\left(t_{0}\right), y^{(1)}\left(t_{0}\right), \ldots, y^{(k-1)}\left(t_{0}\right)\right)[2$. By analyticity, if system (1) satisfies the accessibility rank condition, then the observability rank condition is also equivalent to a local (weak) observability assumption (see [14] for more detail). Note that a notion of (local) observability is needed for the construction of any (local) state observer, and thus is not restrictive.

\section{MAIN RESUlTS}

This section contains our main technical result. Before proceeding to its statement, some facts concerning analytic nonlinear systems are recalled for the convenience of the reader.

Consider the system (1) and fix a point $x \in \mathcal{X}$. By assumption, the flow $\phi_{t}(x)$ of the vector field $f$ is well-defined for all $t \in \mathbb{R}$. Then, the Taylor series expansions of the (analytic) function $h \circ \phi_{t}(x)$ about $t=0$ is given by

$$
h \circ \phi_{t}(x)=\sum_{k=0}^{\infty} L_{f}^{k} h(x) \frac{t^{k}}{k !} .
$$

The above power series expansion, without loss of generality, can be assumed to have radius of convergence $\rho>0$, and to converge on the open interval $(-\rho, \rho)$. Similar considerations apply to the Taylor series expansions of $d h \circ \phi_{t}(x)$ about $t=0$, which is given by

$$
d h \circ \phi_{t}(x)=\sum_{k=0}^{\infty} d L_{f}^{k} h(x) \frac{t^{k}}{k !},
$$

and also converges on the open interval $(-\rho, \rho)$. As a consequence, if $t_{i} \in(-\rho, \rho)$, then

$$
d h \circ \phi_{t_{i}}(x)=\sum_{k=0}^{\infty} d L_{f}^{k} h(x) \frac{t_{i}^{k}}{k !} .
$$

In particular, setting

$$
t_{i}=(i-1) T,
$$

for all $i \in[1, n]$, the expressions in 4 are simultaneously valid for all $T \in I$, with

$$
I=\left(-\frac{\rho}{n-1}, \frac{\rho}{n-1}\right) .
$$

We are now in the position to state our main result.

Theorem 1. Consider the analytic nonlinear system (1) and the mapping $H_{T}$ defined in 2). Suppose that the observability rank condition (3) holds at some given point $x_{0} \in \mathcal{X}$. Then, there exist an open neighbourhood $\mathcal{X}_{0} \subset \mathcal{X}$ of $x_{0}$ such that the restriction of the

${ }^{1}$ Note that for nonlinear systems it is possible to define several, nonequivalent, notions of observability. mapping $H_{T}$ to $\mathcal{X}_{0}$ is a diffeomorphism (onto its image) for generic values of $T \in I$.

Proof. To prove the claim, it is sufficient to show that the Jacobian matrix of $H_{T}$ is non-singular at $x_{0}$ for generic values of $T \in I$, i.e. that the set

$$
\left\{T \in I: \operatorname{rank} H_{T}\left(x_{0}\right)=n\right\}
$$

is an open and dense subset of $I$.

To begin with, since the observability rank condition is satisfied at $x_{0}$, there exist analytic functions $\tilde{h}_{1}, \tilde{h}_{2}, \ldots, \tilde{h}_{n} \in \mathcal{O}$ the differentials of which are linearly independent at $x_{0}$. Thus, each differential of the form $d L_{f}^{k} h\left(x_{0}\right)$, with $k$ a non-negative integer, can be written as

$$
d L_{f}^{k} h\left(x_{0}\right)=\sum_{j=1}^{n} a_{k j}\left(x_{0}\right) d \tilde{h}_{j}\left(x_{0}\right),
$$

in which the $a_{k j}\left(x_{0}\right)$ 's are real coefficients depending on the point $x_{0}$. Now let $t_{i}=(i-1) T$, with $i \in[1, n]$ and $T \in I$. Combining the expression in (4) with that given in (5) yields

$$
\begin{aligned}
d h \circ \phi_{t_{i}}\left(x_{0}\right) & =\sum_{k=0}^{\infty} d L_{f}^{k} h\left(x_{0}\right) \frac{t_{i}^{k}}{k !} \\
& =\sum_{k=0}^{\infty}\left(\sum_{j=1}^{n} a_{k j}\left(x_{0}\right) d \tilde{h}_{j}\left(x_{0}\right)\right) \frac{t_{i}^{k}}{k !} \\
& =\sum_{j=1}^{n}\left(\sum_{k=0}^{\infty} a_{k j}\left(x_{0}\right) \frac{t_{i}^{k}}{k !}\right) d \tilde{h}_{j}\left(x_{0}\right) \\
& =\sum_{j=1}^{n} A_{i j}\left(x_{0}\right) d \tilde{h}_{j}\left(x_{0}\right) .
\end{aligned}
$$

Note that the convergence of the series

$$
\sum_{k=0}^{\infty} a_{k j}\left(x_{0}\right) \frac{t_{i}^{k}}{k !}
$$

with $j \in[1, n]$, can be proved by combining (5) with the assumption of analyticity of the functions $L_{f}^{k} h$ and $\tilde{h}_{j}$. This implies that the Jacobian matrix of the mapping $H_{T}$ at $x_{0}$ can be written as

$$
\frac{\partial H_{T}}{\partial x}\left(x_{0}\right)=A_{T}\left(x_{0}\right) \tilde{H}\left(x_{0}\right)
$$

in which the matrices $A_{T}\left(x_{0}\right) \in \mathbb{R}^{n \times n}$ and $\widetilde{H}\left(x_{0}\right) \in \mathbb{R}^{n \times n}$ are defined as

$$
\begin{aligned}
A_{T}\left(x_{0}\right) & =\left[\sum_{k=0}^{\infty} a_{k j}\left(x_{0}\right) \frac{t_{i}^{k}}{k !}\right]_{i, j=1, \ldots, n}, \\
\widetilde{H}\left(x_{0}\right) & =\left[d \tilde{h}_{i}\left(x_{0}\right)\right]_{i=1, \ldots, n} .
\end{aligned}
$$

It is now sufficient to observe that the set

$$
\left\{T \in I: \operatorname{rank} H_{T}\left(x_{0}\right)<n\right\},
$$

which by the observability assumption on the system coincides with the set

$$
\left\{T \in I: \operatorname{det} A_{T}\left(x_{0}\right)=0\right\},
$$

is the zero set of the analytic function $T \mapsto \operatorname{det} A_{T}\left(x_{0}\right)$, and so is an analytic subset of $I$. Thus, its complement

$$
\left\{T \in I: \operatorname{rank} H_{T}\left(x_{0}\right)=n\right\},
$$

is an open and dense subset of $I$, hence the claim.

From a practical point of view, Theorem 1 has useful consequences. Recalling that the mapping $H_{T}$ gives the delay coordinates vector $Y_{T}$ when evaluated along the trajectories of the system, Theorem 1 
establishes that the trajectories of the delay coordinates vector $Y_{T}$ coincide (up to a local diffeomorphism) with the state trajectories of the system for generic values of the sampling period $T \in I$. In other words, under the stated assumptions, the delay coordinate vector evolves on a manifold that is (locally) diffeomorphic to the original state space for generic values of the parameter $T \in I$. This fact can be used to reconstruct the state of the system from delayed versions of the output signal. Thus, delay coordinates may be an advantageous alternative for practical applications to the commonly used high-order time derivatives of the output signal, which in practice have to be numerically approximated and are significantly affected by noise [15].

Remark 1. A direct by-product of Takens' theorem [12] is that a vector constructed with (at least) $2 n+1$ delayed versions of the output of a $\left(C^{2}\right)$ dynamical system of the form 11 evolves generically $\left.\right|^{2}$ on an embedded submanifold of the state space of the system. In that context, only a mild regularity assumption on the output function is made to obtain a global result. In our framework, by enforcing a (local) observability assumption and a stronger regularity condition (i.e. analiticity), only $n$ delayed versions of the output of the system are needed to form a coordinates vector lying on a state space (locally) diffeomorphic to the original one for generic values of the sampling period on a given set.

\section{An ILlustrative EXAMPLE}

To illustrate the result as well as evaluate the performance of our approach, a simple academic example is considered.

Consider an analytic, nonlinear, system described by equations of the form

$$
\dot{x}_{1}=x_{2}^{2}, \quad \dot{x}_{2}=x_{3}, \quad \dot{x}_{3}=0, \quad y=x_{1},
$$

in which $x(t)=\left(x_{1}(t), x_{2}(t), x_{3}(t)\right) \in \mathbb{R}^{3}, y(t) \in \mathbb{R}$, and the initial condition of the state is set to $x(0)=x^{\star} \in \mathbb{R}^{3} \backslash\{0\}$. The system satisfies the observability rank condition at any point of $\mathbb{R}^{3}$ except for $x=0$. Thus, the following considerations hold as long as the state of the system evolves away from the origin.

The special form of equations (6) permits a direct analysis. A closed form solution of the flow of the system is determined by solving the three differential equations in (6) sequentially, bottom to top. The solution is given by

$$
\begin{aligned}
& x_{1}(t)=x_{1}^{\star}+\left(x_{2}^{\star}\right)^{2} t+x_{3}^{\star} x_{3}^{\star} t^{2}+\frac{1}{3}\left(x_{3}^{\star}\right)^{2} t^{3} \\
& x_{2}(t)=x_{2}^{\star}+x_{1}^{\star} t, \\
& x_{3}(t)=x_{3}^{\star} .
\end{aligned}
$$

Finding an expression of the components of the delay coordinates vector $Y_{T}$ as a function of the components of the state of the system is now an algebraic exercise. The computations leading to such expression can be performed through the use of symbolic computation software. It turns out that

$$
\begin{aligned}
y(t) & =x_{1}(t), \\
y(t+T) & =x_{1}(t)-\frac{1}{3} T^{3} x_{3}^{2}(t)+T^{2} x_{2}(t) x_{3}(t)-T x_{2}^{2}(t), \\
y(t+2 T) & =x_{1}(t)-\frac{8}{3} T^{3} x_{3}^{2}(t)+4 T^{2} x_{2}(t) x_{3}(t)-2 T x_{2}^{2}(t) .
\end{aligned}
$$

Thus, it can be inferred that the mapping $H_{T}$ associated with the system $\sqrt{6}$ is of the form

$$
H_{T}(x)=\left[\begin{array}{c}
x_{1} \\
x_{1}-\frac{1}{3} T^{3} x_{3}^{2}+T^{2} x_{2} x_{3}-T x_{2}^{2} \\
x_{1}-\frac{8}{3} T^{3} x_{3}^{2}+4 T^{2} x_{2} x_{3}-2 T x_{2}^{2}
\end{array}\right],
$$

\footnotetext{
${ }^{2}$ In this case, the topological notion of genericity refers to the output function as an element of the function space of $C^{2}$ maps endowed with the $C^{1}$ topology.
}

for all $x=\left(x_{1}, x_{2}, x_{3}\right) \in \mathbb{R}^{3}$. It is worth noting that, for every fixed $x \in \mathbb{R}^{3}$, the components of the mapping $H_{T}$ are analytic functions of $T$ on the whole real line. The Jacobian matrix of this mapping is given by the expression

$$
\frac{\partial H_{T}}{\partial x}=\left[\begin{array}{ccc}
1 & 0 & 0 \\
1 & T^{2} x_{3}-2 T x_{2} & -\frac{2}{3} T^{3} x_{3}+T^{2} x_{2} \\
1 & 4 T^{2} x_{3}-4 T x_{2} & -\frac{16}{3} T^{3} x_{3}+4 T^{2} x_{2}
\end{array}\right]
$$

for all $x \in \mathbb{R}^{3}$. For every fixed $x \in \mathbb{R}^{3} \backslash\{0\}$, its determinant is a real polynomial of degree three in the unknown $T$, and its zero set is given by

$$
\mathcal{Z}_{x}=\left\{0,\left(\frac{3+\sqrt{3}}{2}\right) \frac{x_{2}}{x_{3}},\left(\frac{3-\sqrt{3}}{2}\right) \frac{x_{2}}{x_{3}}\right\} .
$$

Therefore $H_{T}$ defines a (local) diffeomorphism only if the point $x$ does not lie on either of the hyperplanes defined by

$$
(3 \pm \sqrt{3}) x_{2}+2 T x_{3}=0 .
$$

A consequence of this fact is that, for each value of the sampling period $T \in \mathbb{R}$ and for each initial condition $x^{\star} \in \mathbb{R}^{3} \backslash\{0\}$, there are at most two positive time instants at which the state crosses these hyperplanes. In particular, such time instants are given by

$$
t_{1,2}^{\star}=\frac{-(3 \pm \sqrt{3}) x_{1}^{\star}}{(3 \pm \sqrt{3}) x_{2}^{\star}+2 T x_{3}^{\star}} .
$$

Note that, away from the origin, these hyperplanes are not invariant. This validates the results in Theorem 1 and shows that, in general, the result is conservative. As a matter of fact the mapping $H_{T}$ may (locally) define a diffeomorphism everywhere except for a finite set of values of $T$.

\section{Conclusions}

The problem of local reconstruction of the state of a nonlinear system has been studied. A special mapping, induced by the $n$ dimensional delay coordinates vector and parameterized by the sampling period, has been studied to solve this problem. Under analyticity and (local) rank observability assumptions, the mapping has been shown to define a (local) diffeomorphism for generic values of the sampling period. Some aspects of the proposed methodology have been illustrated on a small academic example.

\section{REFERENCES}

[1] H. Nijmeijer and A. Van der Schaft, Nonlinear Dynamical Control Systems. London, U.K.: Springer-Verlag, 1990.

[2] A. Isidori, Nonlinear control systems (3rd edition). New York: SpringerVerlag, 1995.

[3] J. P. Gauthier and I. Kupka, Deterministic observation theory and applications. Cambridge, U.K.: Cambridge University Press, 2001.

[4] F. Esfandiari and H. K. Khalil, "Output feedback stabilization of fully linearizable systems," Int. J. Control, vol. 56, no. 5, pp. 1007-1037, 1992.

[5] J. M. Coron, "On the stabilization of controllable and observable systems by an output feedback law," Math. Control, Signal, Syst., vol. 7, no. 3, pp. 187-216, 1994.

[6] A. Astolfi and R. Ortega, "Immersion and invariance: a new tool for stabilization and adaptive control of nonlinear systems," IEEE Trans. Autom. Control, vol. 48, no. 4, pp. 590-606, 2003.

[7] G. Besançon, Nonlinear observers and applications. Berlin, Germany: Springer-Verlag, 2007.

[8] V. Andrieu, L. Praly, and A. Astolfi, "Homogeneous approximation, recursive observer design, and output feedback," SIAM J. Control Optim., vol. 47, no. 4, pp. 1814-1850, 2008.

[9] A. Teel and L. Praly, "Tools for semiglobal stabilization by partial state and output feedback," SIAM J. Control Optim., vol. 33, no. 5, pp. $1443-$ $1488,1995$. 
[10] S. Diop, J. W. Grizzle, P. E. Moraal, and A. Stefanopoulou, "Interpolation and numerical differentiation for observer design," in Proc. Amer. Control Conf., Baltimore, MD, 1994, pp. 1329-1333.

[11] D. Aeyels, "Generic observability of differentiable systems," SIAM J. Control Optim., vol. 19, no. 5, pp. 595-603, 1981.

[12] F. Takens, "Detecting strange attractors in turbulence," in Dynamical Systems and Turbulence, ser. Lecture Notes Math., D. Rand and L. S. Young, Eds. Berlin, Germany: Springer-Verlag, 1981, vol. 898, pp. 366-381.

[13] T. Sauer, J. A. Yorke, and M. Casdagli, "Embedology," J. Stat. Phys., vol. 65, no. 3-4, pp. 579-616, 1991.

[14] E. D. Sontag, "A concept of local observability," Syst. Control Lett., vol. 5, no. 1, pp. 41-47, 1984.

[15] H. K. Khalil, Nonlinear systems. Upper Saddle River, NJ: Prentice Hall, 2002. 